\title{
Avaliação do Gradiente Pressórico Aorto-Radial em Pacientes Submetidos à Intervenção Cirúrgica com Circulação Extracorpórea*
}

\section{Evaluation of the Aorta-to-Radial Artery Pressure Gradient in Patients Undergoing Surgery with Cardiopulmonary Bypass}

Maria José Carvalho Carmona, TSA ${ }^{1}$, Luiz Carlos de Melo Barboza Júnior², Roberto Yara Buscattí, Fábio Antônio Gaiotto ${ }^{4}$, Alexandre Ciappina Hueb5, Luiz Marcelo Sá Malbouisson, TSA ${ }^{6}$

\section{RESUMO}

Carmona MJC, Barboza Jr LCM, Buscatti RY, Gaiotto FA, Hueb AC, Malbouisson LMS - Avaliação do Gradiente Pressórico Aorto-Radial em Pacientes Submetidos à Intervenção Cirúrgica com Circulação Extracorpórea.

JUSTIFICATIVA E OBJETIVOS: Diversos estudos têm demonstrado diferença significativa entre a pressão aórtica e a pressão radial em pacientes submetidos à circulação extracorpórea (CEC). Os objetivos deste estudo foram avaliar o comportamento da diferença entre pressão arterial radial e a pressão aórtica durante revascularização do miocárdio (RM) com CEC e sua correlação com resistência vascular sistêmica.

MÉTODO: Após aprovação pelo Comitê de Ética Hospitalar, 16 pacientes submetidos à RM com CEC hipotérmica foram estudados. Pressões sistólica, diastólica e média foram obtidas na raiz da aorta e na artéria radial, através de cateteres específicos. Débito cardíaco foi obtido com o uso de cateter de artéria pulmonar ou diretamente da máquina de $C E C$ e resistência vascular sistêmica indexada (RVSi) foi calculada nos momentos pré-CEC, início da CEC, após a última RM, no fim da CEC e pós-CEC. A análise estatística foi realizada por meio de Análise de Variância para medidas repetidas e correlação de ordem de Spearman e o nível de significância foi fixado em 0,05.

\footnotetext{
*Recebido do (Received from) Hospital das Clínicas da Faculdade de Medicina da Universidade de São Paulo (HC-FMUSP), São Paulo, SP

1. Professora Livre-Docente Associada da Disciplina de Anestesiologia da FMUSP; Diretora da Divisão de Anestesia do Instituto Central do HC-FMUSP 2. Aluno da Graduação da FMUSP; Bolsista de Iniciação Científica da FAPESP (Projeto FAPESP 99/11900-3)

3. Bolsista Fundação Zerbini - Especialização em Anestesia e Pós-operatório de Cirurgia Cardíaca e Torácica do Instituto do Coração do HC-FMUSP 4. Cirurgião Cardíaco; Pós-Graduando do Programa de Pós-graduação em Cirurgia Torácica e Cardiovascular da FMUSP

5. Doutor em Ciências pela USP; Médico Assistente da Divisão de Cirurgia do InCor - HC-FMUSP

6. Doutor em Ciências pela USP; Especialista em Medicina Intensiva - AMIB; Médico Supervisor da UTI-Anestesia do Instituto Central do HC-FMUSP
}

Apresentado (Submitted) em 27 de novembro de 2006

Aceito (Accepted) para publicação em 21 de agosto de 2007

Endereço para correspondência (Correspondence to):

Dra. Maria José Carvalho Carmona

Divisão de Anestesia do ICHC

Av. Enéas Carvalho de Aguiar, 255, $8^{\circ}$ andar - Cerqueira César

05403-900 São Paulo, SP

E-mail:maria.carmona@incor.usp.br
RESULTADOS: Após o início da CEC, a pressão arterial radial foi sistematicamente menor que a pressão aórtica em 3 a $5 \mathrm{mmHg}$. Foi observada correlação significativa entre o gradiente médio de pressão aorto-radial e a RVSi somente após a última RM, correspondendo ao aquecimento do paciente ( $R h o=0,67, p=0,009$ ).

CONCLUSÕES: A medida de pressão na arterial radial subestimou sistematicamente a pressão arterial na raiz da aorta após a CEC e a RVSi não forneceu estimativa acurada da magnitude do gradiente de pressão aorto-radial.

Unitermos: CIRURGIA, Cardíaca: circulação extracorpórea, revascularização miocárdica; MONITORAÇÃO: gradiente de pressão aorto-radial, resistência vascular sistêmica.

\section{SUMMARY}

Carmona MJC, Barboza Jr LCM, Buscatti RY, Gaiotto FA, Hueb AC, Malbuisson LMS - Evaluation of the Aorta-to-Radial Artery Pressure Gradient in Patients Undergoing Surgery with Cardiopulmonary Bypass.

BACKGROUND AND OBJECTIVES: Several studies have demonstrated a significant difference between the aortic and radial artery pressures in patients on cardiopulmonary bypass (CPB). The objectives of this study were to evaluate the behavior of the aorta-to-radial artery pressure gradient during myocardial revascularization (MR) with $C P B$ and its correlation with the systemic vascular resistance.

METHODS: After approval by the Ethics Committee of the hospital, 16 patients undergoing MR with hypothermic CPB were studied. Systolic, diastolic and mean blood pressures were obtained at the root of the aorta and in the radial artery by using specific catheters. The cardiac output was obtained using a pulmonary artery catheter or from the CPB equipment, and the systemic vascular resistance index (SVRI) pre-CPB, at the beginning of $C P B$, after the last $M R$, at the end of the $C P B$, and post-CPB was calculated. Statistical analysis was done with Analysis of Variance for repeated measurements and Spearman correlation, and a level of significance of 0.05 was established.

RESULTS: After beginning CPB, the radial artery pressure was systematically lower than the aortic pressure by 3 to $5 \mathrm{mmHg}$. A significant correlation between the mean aorta-to-radial artery pressure gradient and SVRI was observed only in the last MR, corresponding to the rewarming of the patient ( $R h o=0.67, p=0.009$ ).

CONCLUSIONS: Measurement of the radial artery pressure underestimated, systematically, the arterial pressure at the root of the aorta after CPB and the SVRI did not provide an accurate estimate of the magnitude of the aorta-to-radial artery pressure gradient.

Key Words: MONITORING: aorta-to-radial artery pressure gradient, systemic vascular resistance; SURGERY, Cardiac: cardiopulmonary bypass, myocardial revascularization. 


\section{INTRODUÇÃO}

Diversos estudos têm identificado diferença entre a medida da pressão arterial na raiz da aorta e na artéria radial em pacientes submetidos à cirurgia cardíaca, sobretudo durante e após a circulação extracorpórea (CEC). A etiologia da diferença, embora controversa, tem sido atribuída a uma associação entre alterações do estado contrátil das artérias e arteríolas periféricas, à hipotermia e à condição volêmica do paciente. Alguns autores têm sugerido que o decréscimo na resistência vascular do membro superior ou na mão, secundária à vasodilatação, poderia ser responsável pelo gradiente pressórico aorto-radial ${ }^{1-3}$. Baba e col. e Nakayama e col., por outro lado, relataram que o mecanismo responsável pelo aparecimento da diferença pressórica entre a aorta e a artéria radial seria vasoconstrição/vasoespasmo relacionado com a circulação extracorpórea ou fármacos vasopressores ${ }^{4,5}$. Sustentando essa hipótese, Rich e col. e De Hert e col. observaram que o aparecimento desse gradiente estava associado ao início da circulação extracorpórea ${ }^{6,7}$.

Essa diferença entre a pressão atual medida na raiz da aorta, que determina a pressão de perfusão orgânica e a pressão medida na artéria radial, pode resultar em ajustes inadequados na hemodinâmica de pacientes com função cardiovascular comprometida, levando à hipoperfusão orgânica ou a aumentos desnecessários do consumo miocárdico de oxigênio que poderiam ocasionar isquemia miocárdica. A medida da resistência vascular sistêmica fornece uma estimativa indireta da vasoconstrição arterial e arteriolar e que poderia estar correlacionada com o gradiente de pressão aorto-radial e, assim, contribuir para uma melhor adequação do estado hemodinâmico depois da saída de CEC, minimizando o risco.

Os objetivos deste estudo foram avaliar o comportamento da diferença entre a medida da pressão arterial medida na artéria radial e a pressão atual na raiz da aorta antes, durante e depois de CEC hipotérmica em pacientes submetidos à revascularização cirúrgica do miocárdio (RM) e sua correlação com resistência vascular sistêmica em cada momento.

\section{MÉTODOS}

O tamanho da amostra de 16 indivíduos foi calculado considerando-se um poder de teste de $95 \%$ e um nível de significância de $5 \%$. Após aprovação do projeto de pesquisa pela Comissão Científica do Instituto do Coração e pela Comissão de Ética Médica do Hospital das Clínicas da Faculdade de Medicina da USP, foram estudados 16 pacientes submetidos a revascularização do miocárdio sob circulação extracorpórea. Os critérios para inclusão foram: 1) primeira intervenção cirúrgica para revascularização do miocárdio; 2) procedimento eletivo; 3) ausência de doença vascular arterial periférica. Os critérios para exclusão foram: 1) procedimentos cirúrgicos associados, como revascularização e troca valvar na mesma operação; 2) dissecção ou punção prévia da artéria radial a ser cateterizada para o procedimento cirúrgico; 3) presença de aneurisma de ventrículo esquerdo; 4) disfunção importante de ventrículo direito ao ecocardiograma; 5) complicações mecânicas de infarto como insuficiência mitral ou rotura de septo interventricular; 6) portadores de disfunção orgânica associada; 7) disfunção ventricular esquerda com fração de ejeção $<0,35$; 8) curva de pressão arterial achatada ao monitor ou oclusão precoce do cateter; 9) necessidade de suporte circulatório mecânico com balão intra-aórtico.

Cada paciente recebeu midazolam 0,1 a $0,2 \mathrm{mg} \cdot \mathrm{kg}^{-1}$ por via oral como medicação pré-anestésica 30 minutos antes da operação. Após admissão ao centro cirúrgico, os pacientes foram monitorizados com cardioscópio (derivações DII e V5) e oxímetro de pulso utilizando-se um monitor multiparamétrico Siemens modelo SC7000 (Siemens Medical, Berlim, Alemanha). O acesso venoso com cateter de teflon de calibre 16G foi obtido em cada paciente. A monitoração da pressão arterial média foi obtida por punção da artéria radial direita sob anestesia local, utilizando-se cateter de teflon de calibre $20 \mathrm{G}$. Após verificação de fluxo através do cateter, a linha arterial era conectada a um transdutor de pressão descartável Edwards TRUWAVE (Edwards Lifesciences, Irvine, CA, EUA) com precisão de $\pm 1 \mathrm{mmHg}$. A calibração foi realizada à pressão ambiente e à altura da linha axilar média. $A$ indução anestésica foi realizada com midazolam na dose de 0,3 a $0,5 \mathrm{mg} \cdot \mathrm{kg}^{-1}$, fentanil 20 a $30 \mu \mathrm{g} \cdot \mathrm{kg}^{-1}$ e pancurônio 0,1 $\mathrm{mg} \cdot \mathrm{kg}^{-1}$. A anestesia foi mantida com inalação de isoflurano em concentrações variáveis e doses suplementares de fentanil. Durante a CEC, doses suplementares de midazolam e bloqueador neuromuscular foram administrados para manutenção de hipnose e relaxamento muscular. Após a indução anestésica, foi instalado cateter de artéria pulmonar (CCO/SvO ${ }_{2} /$ VIPTM TD catheter; Edwards Healthcare Co., Irvine, CA, USA) através de punção da veia jugular interna direita. Termômetro esofágico e sonda vesical foram também instalados após a indução anestésica. O volume corrente foi ajustado entre 6 e $8 \mathrm{~mL} . \mathrm{kg}^{-1}$ e a freqüência respiratória entre 10 e 14 inspirações por minuto para manter uma $\mathrm{PaCO}_{2}$ entre 30 e $40 \mathrm{mmHg}$ na gasometria arterial. O tempo inspiratório foi mantido em $33 \%$ do tempo total do ciclo respiratório. A pressão expiratória final positiva foi ajustada entre 3 e $5 \mathrm{cmH}_{2} \mathrm{O}$ de maneira a não atrapalhar a visualização do campo cirúrgico.

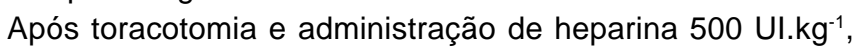
uma cânula arterial dotada de conexão lateral tipo luer-lock na sua porção proximal foi inserida na aorta ascendente e cânulas venosas foram inseridas na veia cava superior e inferior. A circulação extracorpórea foi realizada com fluxo não-pulsátil sob hipotermia moderada utilizando oxigenador de membrana OXI Master Century (Braile, São José do Rio Preto, SP, Brasil), circuito preenchido com solução de Ringer com lactato e $50 \mathrm{~g}$ de manitol a $20 \%$. Após início da CEC, a temperatura central dos pacientes foi reduzida e mantida entre $32^{\circ}$ e $34^{\circ} \mathrm{C}$. O fluxo programado da CEC foi de $100 \mathrm{~mL} . \mathrm{kg}^{-1}$ 
e aferido diretamente do fluxômetro do equipamento de circulação extracorpórea, correspondendo, naquele momento, de forma indireta, ao débito cardíaco do paciente. Após o final das anastomoses, a temperatura central do paciente era elevada até $37^{\circ} \mathrm{C}$ e a assistência circulatória promovida pelo equipamento de circulação extracorpórea era reduzida gradualmente até o retorno pleno da circulação fisiológica. Fármacos vasoativos e vasodilatadores eram infundidos em doses variáveis para facilitar a saída de CEC. Após o término da operação, os pacientes foram transportados para a unidade de terapia intensiva.

Para investigar o impacto da circulação extracorpórea e hipotermia sobre o comportamento temporal do gradiente de pressão entre a aorta e a artéria radial, logo após a instalação da cânula na raiz da aorta, o cirurgião conectou uma linha de monitorização de pressão à conexão lateral da cânula aórtica. Para coleta dos dados hemodinâmicos, os transdutores de pressão arterial radial, aórtica, da linha arterial pulmonar e da pressão de átrio direito foram simultaneamente calibrados. A coleta dos dados hemodinâmicos para a finalidade deste estudo foi realizada nos seguintes momentos: 1) após a colocação da cânula de aorta, antes do início da CEC; 2) antes do pinçamento aórtico, após resfriamento do paciente a $32^{\circ} \mathrm{C}$; 3) após a última anastomose, com temperatura central mantida em $32^{\circ} \mathrm{C}$ e 4) no final da CEC, após reaquecimento do paciente até $37^{\circ} \mathrm{C}$ e antes do início do desmame da CEC e 5) 10 minutos depois da saída de CEC, tendo o paciente reassumido a circulação espontânea, após a infusão de protamina.

Além das medidas de pressão nas artérias radial e aórtica, foram realizadas avaliações da resistência vascular sistêmica indexada (RVSi). Para realização dos cálculos da RVSi, o índice cardíaco foi computado a partir da média de três injeções de solução glicosada a $5 \%$ à temperatura ambiente (em torno de $21^{\circ} \mathrm{C}$ ) por meio da técnica de termodiluição ${ }^{8,9}$ nos momentos pré-CEC e pós-CEC. Durante a CEC, o índice cardíaco foi computado a partir do fluxo total fornecido pelo equipamento de CEC. A drenagem venosa foi ajustada de maneira a se obter uma pressão de valor zero no átrio direito (PAD). A resistência vascular sistêmica indexada foi calculada utilizando a formula seguinte:

$$
\mathrm{RVSi}=\frac{\mathrm{PAM}-\mathrm{PAD}}{\mathrm{IC}} \times 80
$$

A distribuição normal dos dados hemodinâmicos colhidos foi testada utilizando o teste de Kolmogorov-Smirnov. A comparação dos valores da diferença de pressão arterial média, sistólica e da resistência vascular sistêmica ao longo do estudo foi avaliada pela Análise de Variância para medidas repetidas. Concordância no comportamento temporal da diferença de pressão aorto-radial média e da resistência vascular sistêmica indexada foi avaliada pela presença de interação na análise de duas vias para medidas repetidas. Para esse fim, as análises do fator intergrupo (entre RVS e gradiente de pressão aorto-radial) e do fator intragrupo (tempo) foram desconsideradas, sendo levada em consideração apenas a análise de interação. Caso houvesse interação, significando não-concordância entre o comportamento temporal das variáveis estudadas, seriam realizados testes de correlação entre as variáveis nos diversos momentos estudados através do teste de Spearman. O nível de significância foi fixado em 0,05 e as correlações foram consideradas significativas quando o coeficiente Rho foi maior que 0,5 . Os dados estão apresentados como média \pm desvio-padrão.

\section{RESULTADOS}

Os dados referentes ao sexo, idade, peso, altura e superfície corpórea dos pacientes podem ser vistos na tabela I. A figura 1 mostra o comportamento da pressão arterial ao longo dos momentos experimentais. Como pode ser observado, houve diminuição significativa dos valores de pressão arterial média tanto na raiz da aorta quanto na artéria radial, logo após o início da circulação extracorpórea, que foram restabelecidos aos valores iniciais nos momentos seguintes. Como esperado, houve uma redução significativa da temperatura de $35,1 \pm 0,8^{\circ} \mathrm{C}$ para $33 \pm 1^{\circ} \mathrm{C}$ após o início da CEC ( $p<0,001)$ e a temperatura foi normalizada $(36,6 \pm$ $0,7^{\circ} \mathrm{C}$ ) no final da CEC e mantida após a saída de CEC. Em todos os momentos estudados, exceto logo após o início da circulação extracorpórea, a medida de pressão média arterial obtida na raiz da aorta foi menor que a medida obtida na artéria radial (Figura 2). Quando a resistência vascular sistêmica foi estuda, observou-se um comportamento temporal inverso ao comportamento do índice cardíaco (Figura 3) Por outro lado, a resistência vascular sistêmica indexada apresentou variações concordantes com a variação do gradiente pressórico aorto-radial médio, exceto no momento final, após a saída de CEC. Contudo, apenas no momento após a realização da última anastomose no miocárdio foi observada correlação significativa entre a resistência vascular sistêmica indexada e o gradiente de pressão aortoradial $(\mathrm{Rho}=0,67, \mathrm{p}=0,009)$ (Figura 4 )

Tabela I - Dados Demográficos

\begin{tabular}{lc}
\hline Idade $(\text { anos })^{*}$ & $63 \pm 10,40$ \\
Peso $(\mathrm{kg})^{*}$ & $70 \pm 10$ \\
Altura $(\mathrm{m})^{*}$ & $1,66 \pm 0,08$ \\
Superfície corpórea $\left(\mathrm{m}^{2}\right)^{*}$ & $1,82 \pm 0,19$ \\
Sexo & \\
Masculino & 14 \\
Feminino & 2 \\
\hline
\end{tabular}

* Valores expressos em Média \pm DP. 


\section{AVALIAÇÃO DO GRADIENTE PRESSÓRICO AORTO-RADIAL EM PACIENTES SUBMETIDOS À INTERVENÇÃO CIRÚRGICA COM CIRCULAÇÃO EXTRACORPÓREA}
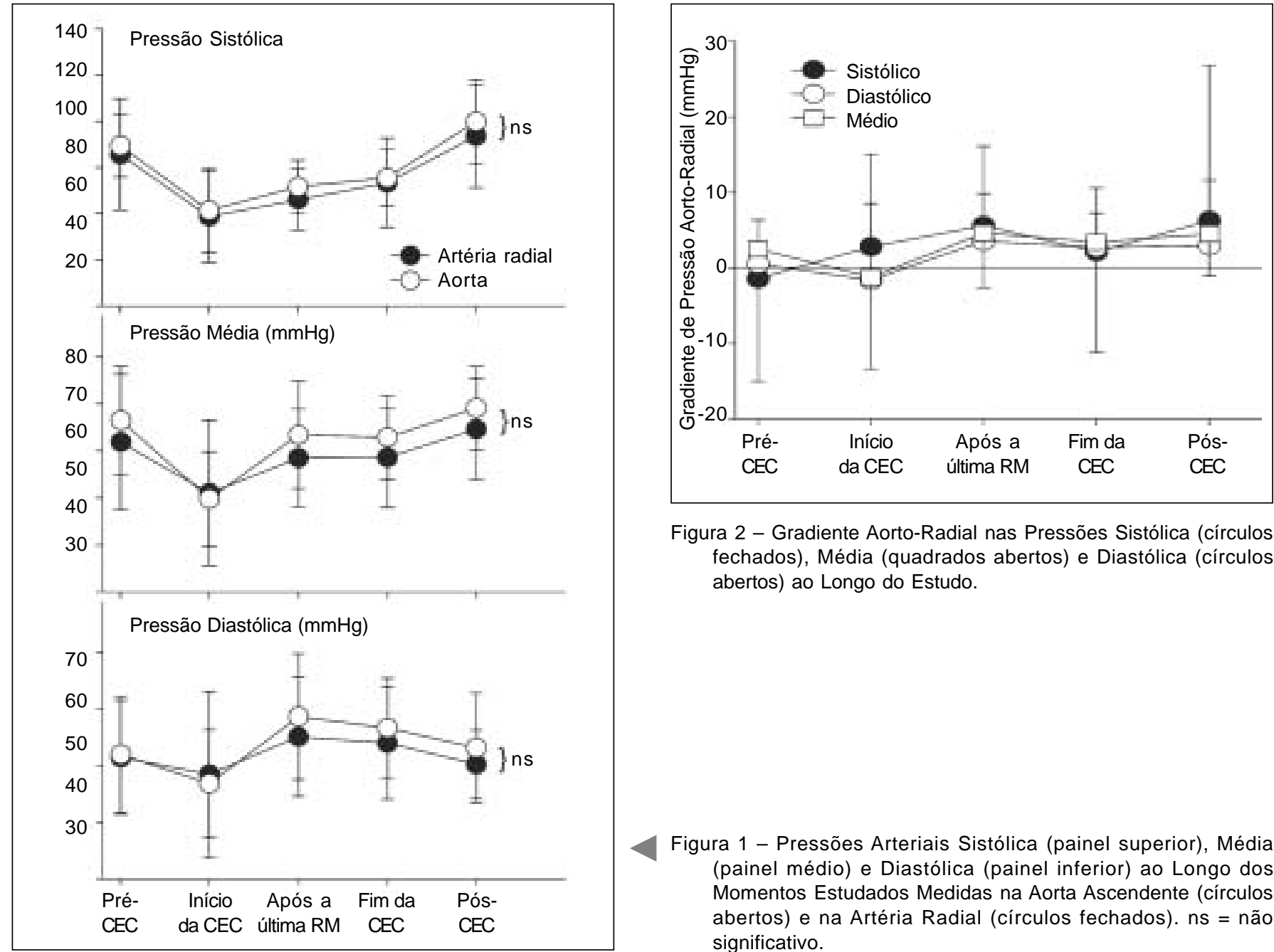

Figura 2 - Gradiente Aorto-Radial nas Pressões Sistólica (círculos fechados), Média (quadrados abertos) e Diastólica (círculos abertos) ao Longo do Estudo.

Figura 1 - Pressões Arteriais Sistólica (painel superior), Média (painel médio) e Diastólica (painel inferior) ao Longo dos Momentos Estudados Medidas na Aorta Ascendente (círculos abertos) e na Artéria Radial (círculos fechados). ns = não significativo.

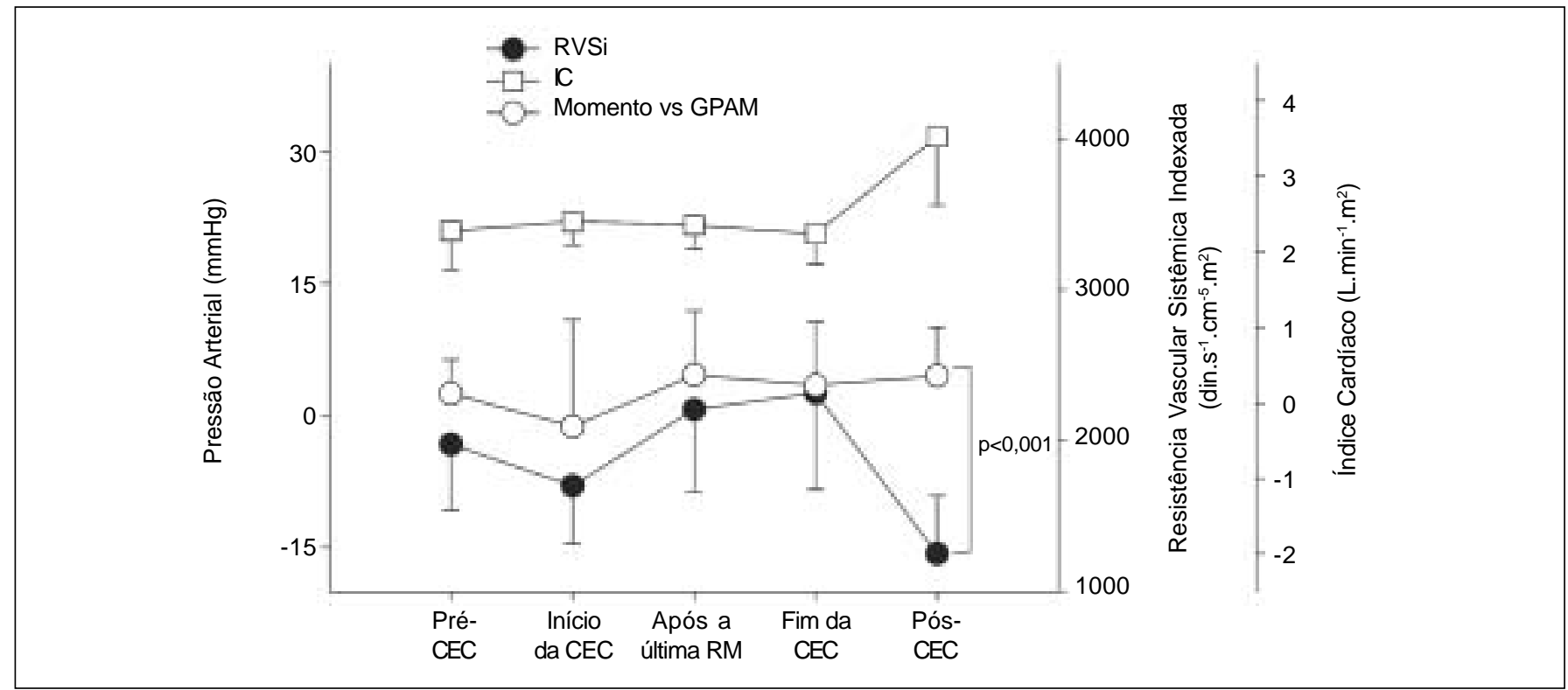

Figura 3 - Comportamento Temporal do Gradiente Aorto-Radial de Pressão Média (círculos abertos), Resistência Vascular Sistêmica Indexada (círculo fechado) e do Índice Cardíaco (quadrado aberto) ao Longo do Estudo. 


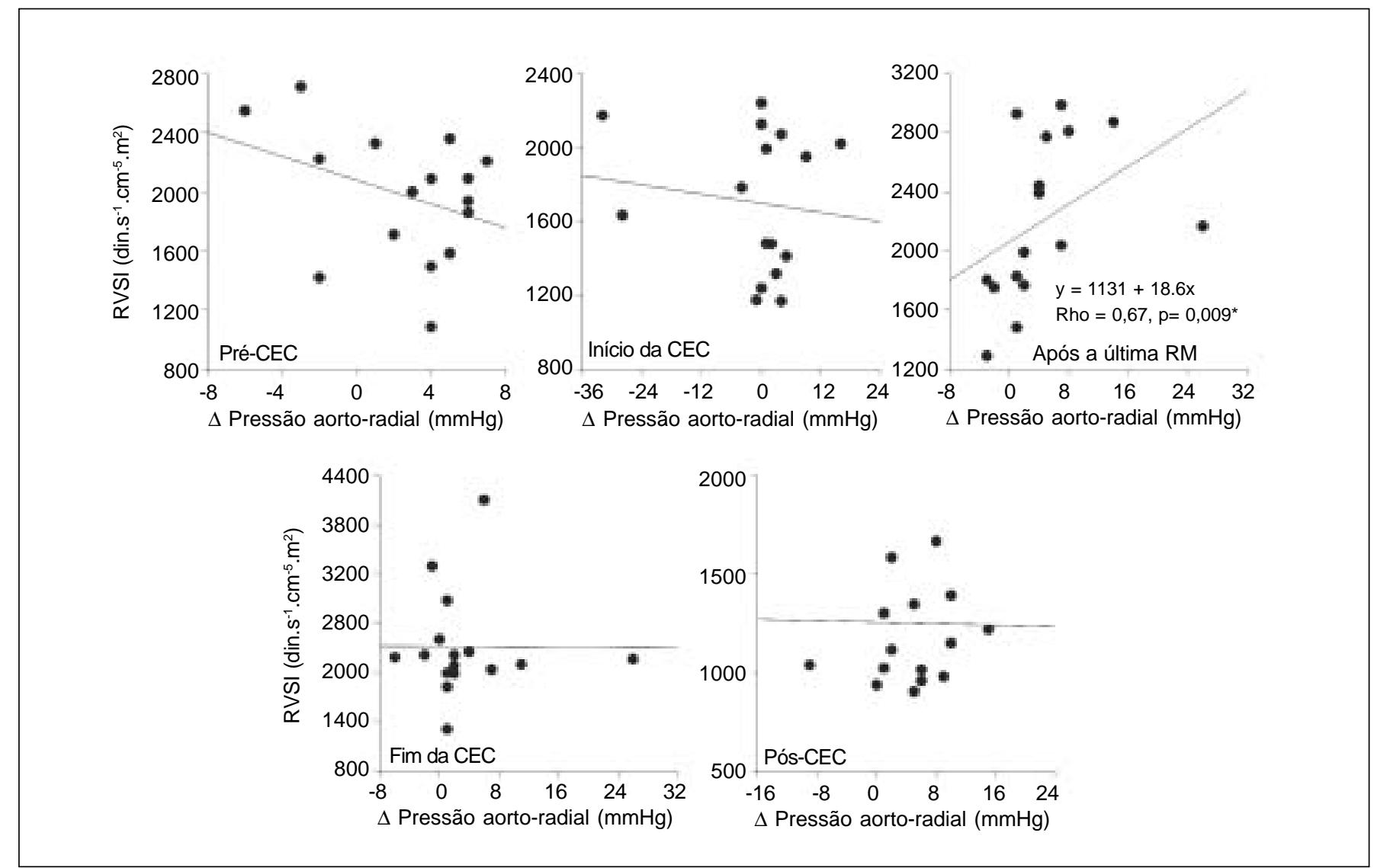

Figura 4 - Gráfico de Dispersão Avaliando a Correlação entre a Resistência Vascular Sistêmica Indexada e o Gradiente Aorto-Radial de Pressão Média nos Momentos Pré-CEC (painel superior esquerdo), Início de CEC (painel superior central), após a Última Revascularização Miocárdica (painel superior direito), ao Final da CEC (painel inferior esquerdo) e após a Saída de CEC (painel inferior direito).

\section{DISCUSSÃO}

Os principais resultados deste estudo foram: 1) a pressão arterial média medida na artéria radial subestima a pressão arterial mensurada na raiz da aorta após o início da CEC; contudo, essa diferença de pressão gira em torno de 3 a 5 $\mathrm{mmHg}$, em valores médios; 2) não houve correlação consistente entre o gradiente pressórico aorto-radial e o índice de resistência vascular sistêmica após a instalação de circulação extracorpórea.

A monitoração invasiva da pressão arterial radial é realizada de forma rotineira em pacientes submetidos à intervenção cirúrgica cardíaca com circulação extracorpórea, em decorrência da necessidade de controle em tempo real da pressão de perfusão orgânica, facilidade de instalação do cateter e baixa incidência de complicações. Com base nos valores da pressão da artéria radial são indicados fármacos vasopressores ou vasodilatadores, com o intuito de ajustar a resistência vascular sistêmica e manter adequado balanço entre o maior débito cardíaco possível e a pressão de perfusão para os órgãos. Nessa população específica de pacientes submetidos a intervenção cirúrgica cardíaca sob circulação extracorpórea hipotérmica, diversos estudos têm demonstrado discrepância entre a medida da pressão na artéria radial e a medida da pressão na raiz da aorta ${ }^{6,10-12}$, o que poderia contribuir para o manuseio inadequado da hemodinâmica, podendo levar ao aumento inapropriado do consumo de oxigênio pelo miocárdio quando a pressão radial subestima a pressão aórtica ou hipoperfusão tecidual em pacientes nos quais a pressão da arterial radial é superior à pressão aórtica ${ }^{6,11}$. Neste estudo, foi observado após o início da CEC que a pressão arterial média na raiz da aorta foi inferior à pressão radial em média $1,2 \mathrm{mmHg}$. Esse gradiente foi invertido nos momentos seguintes, sendo a pressão aórtica média 3 a $5 \mathrm{mmHg}$ maior que a pressão arterial radial média. Em situações nas quais é necessária infusão de altas concentrações de vasopressores, esse gradiente pode ser ainda mais acentuado.

Em virtude da falta de acurácia da medida de pressão arterial radial em refletir a pressão atual na raiz da aorta, outros índices, como a resistência vascular sistêmica, poderiam teoricamente fornecer estimativas da magnitude da diferença entre a pressão aórtica e a pressão radial durante e após a fase hipotérmica da CEC e propiciar avaliações mais adequadas para os ajustes hemodinâmicos necessários. Apesar do comportamento temporal da RVSi ser concordante 
com o gradiente de pressão aorto-radial na maioria dos momentos, como observado na figura 3 , observou-se apenas correlação entre a resistência vascular sistêmica e o gradiente de pressão aorto-radial no momento após a realização da ultima anastomose miocárdica, quando o paciente já estava sendo aquecido e recebendo infusão de fármacos vasoativos. A resistência vascular sistêmica calculada por medidas de hemodinâmica invasiva à beira do leito com cateteres intravasculares é computada como a razão entre a diferença pressórica da arterial radial para pressão no átrio direito e o fluxo sistêmico, refletindo principalmente a impedância promovida pela microcirculação arteriolar e território venoso ao fluxo sangüíneo. A ausência de correlação sugere que outros fatores além desse componente resistivo arteriolar e venoso possam contribuir para a gênese do gradiente pressórico aorto-radial. Contudo, além desse componente resistivo circulatório microvascular, sobretudo após o início da CEC, outros fatores parecem também estar relacionados com a modulação da transmissão de pressão nas artérias, não permitindo inferências da magnitude do gradiente pressórico aorto-radial a partir da RVSi.

Diversos mecanismos têm sido propostos alternativamente para explicar a diferença aorto-radial de pressão em pacientes submetidos à CEC, sendo a hipotermia, a instabilidade hemodinâmica, a mudança do regime de fluxo pulsátil para fluxo contínuo não-pulsátil e a variação do calibre de grandes artérias devido à alteração da elasticidade arterial induzida pela CEC ou pelo uso de altas doses de fármacos vasomoduladores os mais citados. Logo após o início da CEC, a pressão na artéria radial foi discretamente superior à raiz da aorta. Nesse momento, ocorre intensa hemodiluição por causa da entrada na circulação de 2 litros de solução de Ringer com lactato, causando diminuição abrupta do hematócrito e conseqüente redução da viscosidade sangüínea e da resistência vascular sistêmica ${ }^{13}$, o que poderia compensar a vasoconstrição induzida pela hipotermia e pela liberação de mediadores inflamatórios durante a CEC ${ }^{6,7}$. Durante o transcurso da CEC, em geral grande parte do fluido cristalóide constituindo o perfusato extravasa para o espaço extravascular em conseqüência à resposta inflamatória sistêmica induzida pela CEC ${ }^{14}$, o que poderia causar um aumento do hematócrito e viscosidade sangüínea, contribuindo para o aumento da resistência vascular arterial nas artérias de menor calibre, e que justificaria o início e a manutenção do gradiente aorto-radial em valores de 3 a $5 \mathrm{mmHg}$ constantes mesmo após o desmame da CEC ${ }^{15}$. De Hert e col., por outro lado, não observaram correlação entre a magnitude do gradiente de pressão e as alterações observadas no hematócrito e na temperatura sangüínea em uma casuística de 68 pacientes ${ }^{7}$. Outro fator possivelmente relacionado com a gênese do gradiente de pressão aorto-radial seria o calibre das grandes artérias. Estudando 12 pacientes submetidos à intervenção cirúrgica cardíaca com CEC, Kanazawa e col. observaram que sete apresentaram diferença de pressão aorto-radial superior a $10 \mathrm{mmHg}$ após a CEC ${ }^{12}$.
Essa diminuição de pressão na artéria radial em relação à aorta estava associada a uma diminuição progressiva na velocidade da onda de pulso em direção à periferia. Usando a equação proposta por Moens-Korteweg para o cálculo da elasticidade arterial a partir da velocidade da onda de pulso ${ }^{16}$, esses autores computaram uma diminuição na elasticidade, que poderia levar a um amortecimento da onda de pulso e da pressão arterial ao nível radial, explicando o aparecimento do gradiente aorto-radial de pressão ${ }^{12}$. Essa alteração do tônus vascular poderia ser secundária a liberação de substâncias vasoativas, em geral observada após o início da CEC ${ }^{14}$. Finalmente, alterações anatômicas, como os ateromas arteriais, poderiam contribuir para a diminuição da pressão radial em relação à pressão aórtica. Por causa da inversão do gradiente pressórico logo após o início da CEC, sugerindo ausência de estenose arterial, a presença de alterações anatômicas não parece ser fator contributivo importante para a gênese do gradiente aorto-radial neste estudo. Nos casos em que a condição da artéria radial pode ser considerada fator importante na gênese do gradiente, a monitorização pode ser substituída por punção de artéria braquial ou femoral ${ }^{17}$.

Em conclusão, a pressão radial subestimou sistematicamente a pressão na raiz da aorta após a instalação de circulação extracorpórea hipotérmica em pacientes submetidos à revascularização do miocárdio, sendo esse gradiente pressórico de 3 a $5 \mathrm{mmHg}$ neste estudo. Apesar de pequena, essa diferença pode levar a condutas não-apropriadas ou eventualmente deletérias. A resistência vascular sistêmica não permite uma estimativa acurada da magnitude do gradiente de pressão aorto-radial, devendo essa diferença de 3 a $5 \mathrm{mmHg}$ entre a pressão aórtica e a pressão radial ser levada em conta no momento da tomada de decisões terapêuticas em pacientes submetidos à revascularização do miocárdio com CEC hipotérmica.

\section{Evaluation of the Aorta-to-Radial Artery Pressure Gradient in Patients undergoing Surgery with Extracorporeal Circulation}

Maria José Carvalho Carmona, TSA, M.D.; Luiz Carlos de Melo Barboza Júnior; Roberto Yara Buscatti, M.D.; Fábio Antônio Gaiotto, M.D.; Alexandre Ciappina Hueb, M.D.; Luiz Marcelo Sá Malbouisson, TSA, M.D.

\section{INTRODUCTION}

Several studies have identified differences between the arterial pressure measured at the root of the aorta and in the radial artery in patients undergoing cardiac surgery, particularly during and after cardiopulmonary bypass (CPB). The 
etiology of the difference, although controversial, has been attributed to an association between the state of contractility of the peripheral arteries and arterioles, hypothermia and the blood volume of the patient. Some authors have suggested that a reduction in vascular resistance in the upper limb or in the hand, secondary to vasodilation, could be responsible for the aorta-to-radial artery pressure gradient ${ }^{1-3}$. On the other hand, Baba et al. and Nakayama et al. reported that the mechanism responsible for the pressure gradient between the aorta and radial artery could be the vasoconstriction/vasospasm related with the cardiopulmonary bypass or vasopressors ${ }^{4,5}$. To support this hypothesis, Rich et al. and De Hert et al. observed that the development of this pressure gradient was associated with the beginning of the cardiopulmonary bypass ${ }^{6,7}$.

This difference between the pressure measured at the root of the aorta, which determines the perfusion pressure of the body, and the pressure in the radial artery can result in inadequate adjustments in the hemodynamic parameters of patients with compromised cardiovascular function, leading to hypoperfusion and unnecessary increases in myocardial oxygen consumption that could lead to myocardial ischemia. Measurement of the systemic vascular resistance provides an indirect estimate of the arterial and arteriolar vasoconstriction that could be related to the aorta-to-radial artery pressure gradient and, therefore, contribute for a better accommodation of hemodynamic parameters after removal of CPB, minimizing the risk.

The objectives of this study were to evaluate the behavior of the pressure gradient between the radial artery and the root of the aorta before, during, and after hypothermic CPB in patients undergoing surgical myocardial revascularization (MR) and its correlation with the systemic vascular resistance at each moment,

\section{METHODS}

The size of the study population, 16 patients, was determined considering a test power of $95 \%$ and a level of significance of $5 \%$. After approval of the research project by the Scientific Commission of the Instituto do Coração and by the Medical Ethics Commission of the Hospital das Clínicas da Faculdade de Medicina da USP, 16 patients undergoing myocardial revascularization with cardiopulmonary bypass were enrolled. Inclusion criteria were: 1) first myocardial revascularization; 2) elective surgery; and 3) absence of peripheral artery disease. Exclusion criteria included: 1) associated surgeries, such as revascularization and change of valve in the same surgery; 2) dissection or prior puncture of the radial artery that supposed to be catheterized during the surgery; 3 ) presence of aneurysm of the left ventricle; 4) electrocardiographic evidence of important right ventricular dysfunction; 5) infarct-related mechanical complications, such as mitral regurgitation or rupture of the interventricular septum; 6) presence of associated organic dysfunction: 7) left ventricular dysfunction with ejection fraction $<0.35$; 8) blunted arterial pressure curve on the monitor or early occlusion of the catheter; and 9) need of mechanical circulatory support with aortic balloon counterpulsation.

Each patient was medicated with oral midazolam, 0.1 to $0.2 \mathrm{mg} . \mathrm{kg}^{-1} 30$ minutes before the surgery. After admission to the operating room, patients were monitored with a cardioscope (DII and V5 derivations) and pulse oximetry with a multiparametric Siemens monitor model SC7000 (Siemens Medical, Berlin, Germany). Venipuncture with a 16G teflon catheter in every patient. To monitor the mean arterial pressure a $20 \mathrm{G}$ teflon catheter was introduced in the right radial artery. After confirmation of the proper positioning by the backflow of blood through the catheter, the arterial line was connected to a disposable Edwards TRUWAVE pressure transducer (Edwards Lifesciences, Irvine, CA, USA) with precision of $\pm 1 \mathrm{mmHg}$. Calibration was done at ambient pressure, and at the level of the middle axillary line. Induction of anesthesia was accomplished with midazolam, 0.3 to $0.5 \mathrm{mg} \cdot \mathrm{kg}^{-1}, 20$ to $30 \mu \mathrm{g} . \mathrm{kg}^{-1}$ of fentanyl and $0.1 \mathrm{mg} \cdot \mathrm{kg}^{-1}$ of pancuronium. Anesthesia was maintained with variable concentrations of inhalational isoflurane and supplementary doses of fentanyl. During the CPB, supplementary doses of midazolam and neuromuscular blocker were administered to maintain hypnosis and muscular relaxation. After induction, a pulmonary artery catheter (CCO/SvO2/VIPTM TD catheter, Edwards Healthcare Co., Irvine, CA, USA) was introduced in the right internal jugular vein. An esophageal thermometer and bladder catheter were also introduced after anesthetic induction. Tidal volume was set between 6 and $8 \mathrm{~mL} . \mathrm{kg}^{-1}$ and respiratory rate between 10 and 14 inspirations per minute to maintain $\mathrm{PaCO}_{2}$ between 30 and $40 \mathrm{mmHg}$. Inspiration was set as $33 \%$ of the respiratory cycle. Positive end-expiratory pressure was set between 3 and $5 \mathrm{~cm} \mathrm{H}_{2} \mathrm{O}$ to avoid hindering visualization of the surgical field.

After thoracotomy and administration of $500 \mathrm{IU} . \mathrm{kg}^{-1}$ of heparin, an arterial cannula with a lateral luer-lock connection at the proximal end was inserted in the ascending aorta and venous cannulas were introduced in the superior and inferior vena cava. Cardiopulmonary bypass was performed with a non-pulsatile flow under moderate hypothermia using a membrane oxygenator OXI Master Century (Braile, São José do Rio Preto, SP, Brazil), the circuit was filled with Ringer's lactate and $50 \mathrm{~g}$ of $20 \%$ mannitol. After starting the CPB, the core temperature of the patient was reduced and maintained between $32^{\circ}$ and $34^{\circ} \mathrm{C}$. The programmed flow of the CPB was $100 \mathrm{~mL} . \mathrm{kg}^{-1}$ and was checked directly from the flow meter of the cardiopulmonary bypass equipment corresponding indirectly to the cardiac output of the patient. After the end of the anastomosis, the core temperature of the patient was elevated to $37^{\circ} \mathrm{C}$ and the cardiocirculatory assistance of the cardiopulmonary bypass equipment was gradually reduced until full return of the physiological circulation. Variable doses of vasoactive and vasodilator drugs were infused to facilitate removal of the CPB. After the end of the surgery, patients were transported to the intensive care unit. 


\section{EVALUATION OF THE AORTA-TO-RADIAL ARTERY PRESSURE GRADIENT IN PATIENTS UNDERGOING SURGERY WITH CARDIOPULMONARY BYPASS}

To investigate the impact of the cardiopulmonary bypass and hypothermia on the temporal behavior of the aorta-to-radial artery pressure gradient, immediately after insertion of the cannula in the root of the aorta, the surgeon connected a pressure monitoring device to the lateral connection of the aortic cannula. To collect hemodynamic data, the transducers of radial and aortic pressures, arterial pulmonary pressure, and right atrial pressure were calibrated simultaneously. Hemodynamic data were recorded at the following times: 1) after placement of the aortic catheter, before beginning CPB; 2) before occlusion of the aorta, after cooling the patient to $32^{\circ} \mathrm{C}$; 3) after the last anastomosis, with core temperature at $32^{\circ} \mathrm{C}$; 4) at the end of $\mathrm{CPB}$, after rewarming the patient to $37^{\circ} \mathrm{C}$ and before removing the CPB; and 5) 10 minutes after removal of the CPB, after the patient had resumed spontaneous circulation, and after the administration of protamine. Besides measurement of radial artery and aortic pressures, the systemic vascular resistance index (SVRI) was evaluated. To calculate the SVRI the cardiac index was determined as the mean of three injections of D5W at room temperature (around $21^{\circ} \mathrm{C}$ ) by thermodilution ${ }^{8,9}$ pre- and post-CPB. During $\mathrm{CPB}$, the cardiac index was determined from the total flow provided by the CPB equipment. Venous drainage was adjusted in order to obtain a pressure of zero in the right atrium (RAP). The systemic vascular resistance index was calculated according to the following formula:

$$
S V R I=\frac{P A M-P A D}{C l} \times 80
$$

The normal distribution of the hemodynamic data gathered was determined with the Kolmogorov-Smirnov test. Comparison of the difference of mean and systolic blood pressure, and of the systemic vascular resistance throughout the study was done by Analysis of Variance for repeated measurements. Concordance of the temporal behavior of the aortato-radial artery pressure gradient and systemic vascular resistance index was evaluated by the presence of interaction in the two-way analysis of repeated measurements. For this purpose, only the interaction analysis, and not the analysis of intergroup factors (between SVR and aorta-to-radial artery pressure gradient) and intragroup (time), was considered. If interaction were present, i.e., the absence of concordance in the temporal behavior of the parameters, correlation tests among the parameters at different moments were conducted using the Spearman test. The level of significance was set at 0.05 and the correlations were considered significant when the Rho coefficient was greater than 0.5. Data are presented as mean \pm SD.

\section{RESULTS}

Table I shows the data regarding gender, age, weight, height, and body surface area. Figure 1 shows the behavior of the blood pressure at the different moments of the experiment.
Table I - Demographic Data

\begin{tabular}{lc} 
Age $(\text { years })^{*}$ & $63 \pm 10,40$ \\
Weight $(\mathrm{kg})^{*}$ & $70 \pm 10$ \\
Height $(\mathrm{m})^{\star}$ & $1.66 \pm 0.08$ \\
Body surface area $\left(\mathrm{m}^{2}\right)^{*}$ & $1.82 \pm 0.19$ \\
Gender & \\
Male & 14 \\
Female & 2 \\
\hline
\end{tabular}

* Values expressed as Mean \pm SD.

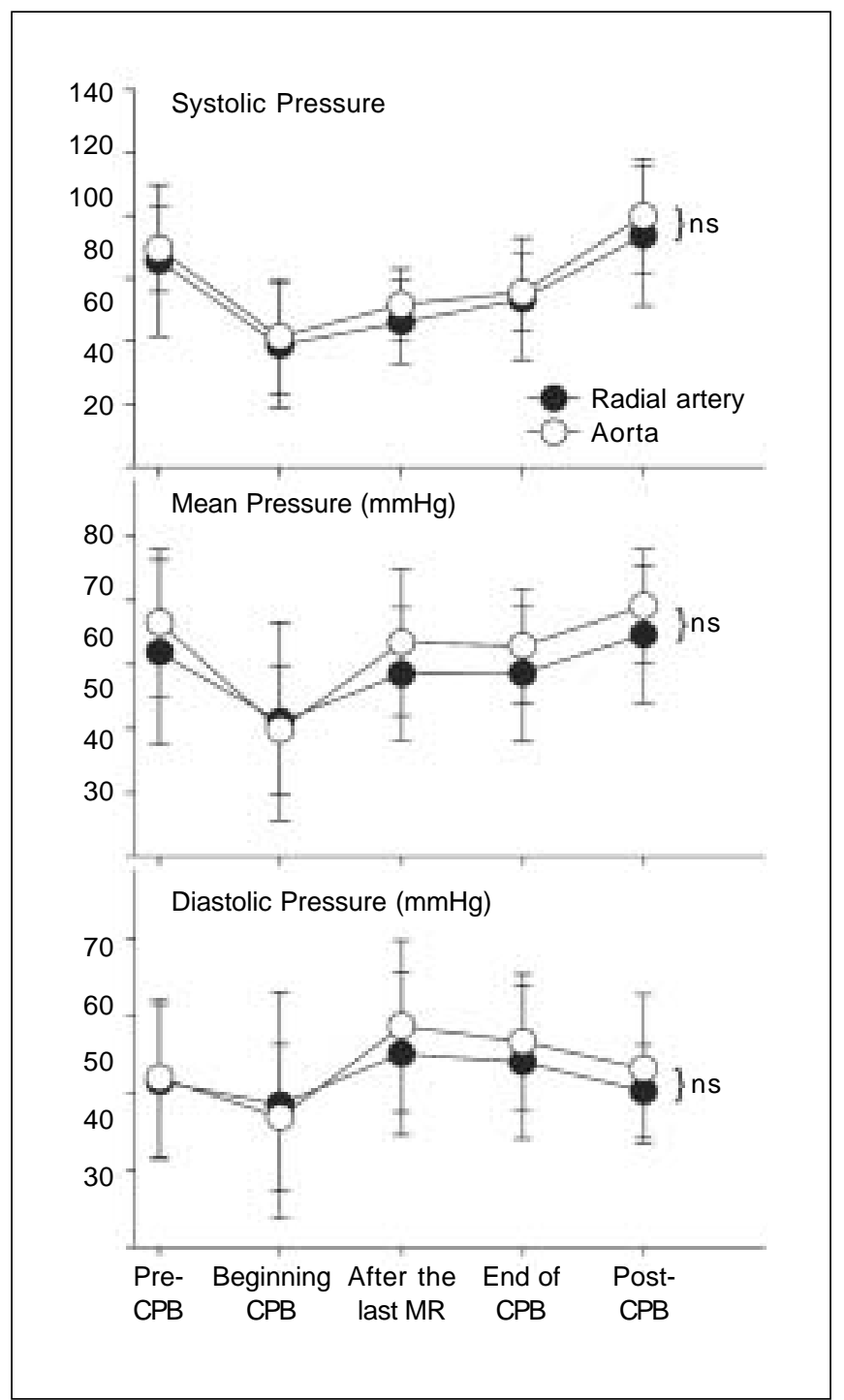

Figure 1 - Systolic (upper panel), Mean (middle panel), and Diastolic (lower panel) Pressures in the Ascending Aorta (open circles) and Radial Artery (closed circles) at Different Moments of the Surgery. ns = non-significant. 
As can be observed there was a significant reduction in mean arterial pressure, both at the root of the aorta and in the radial artery, immediately after starting CPB, returning to normal afterwards. As it was expected, there was a significant reduction in body temperature from $35.1 \pm 0.8^{\circ} \mathrm{C}$ to $33 \pm 1^{\circ} \mathrm{C}$ after the institution of the CPB $(p<0.001)$; temperature brought to normal values $\left(36.6 \pm 0.7^{\circ} \mathrm{C}\right)$ at the end of $\mathrm{CPB}$, which was maintained after discontinuation of CPB. The mean arterial pressure at the root of the aorta was smaller than in the radial artery in all moments of the study except immediately after the initiation of the CPB (Figure 2). The temporal behavior of the systemic vascular resistance was opposite of the cardiac index (Figure 3). On the other hand, the systemic vascular resistance index showed variations in accordance with the variation of the mean aorta-to-radial artery pressure gradient, except for the final moment, after discontinuation of CPB. However, only after the last anastomosis in the myocardium a significant correlation between the systemic vascular resistance index and the aorta-to-radial artery pressure gradient was observed $(\mathrm{Rho}=0.67, \mathrm{p}=0.009)$ (Figure 4).

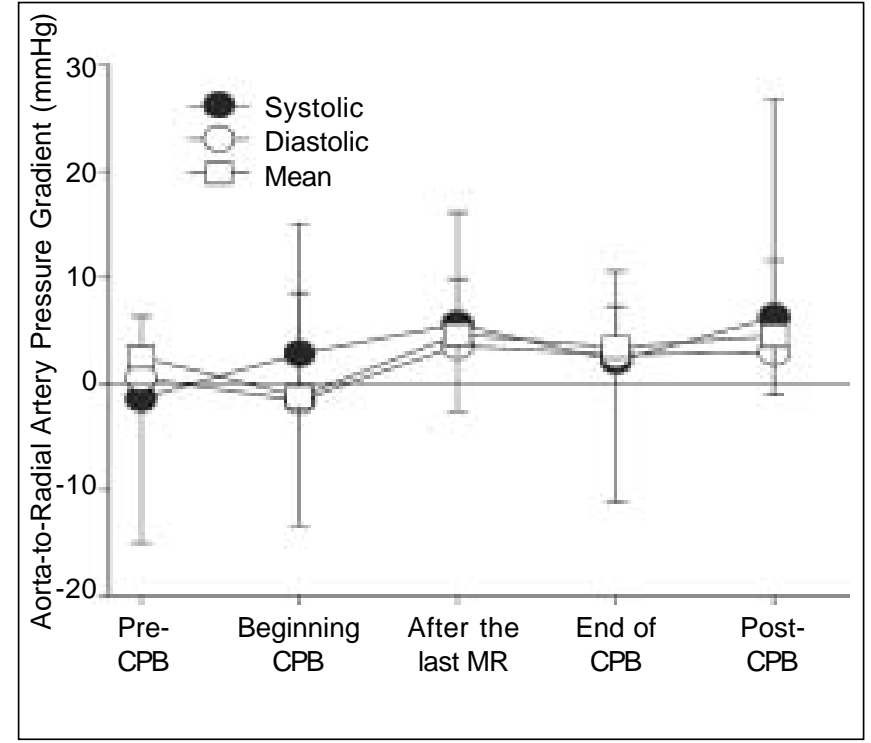

Figure 2 - Systolic (closed circles), Mean (open squares), and Diastolic (open circles) Aorta-to-Radial Artery Pressure Gradients.

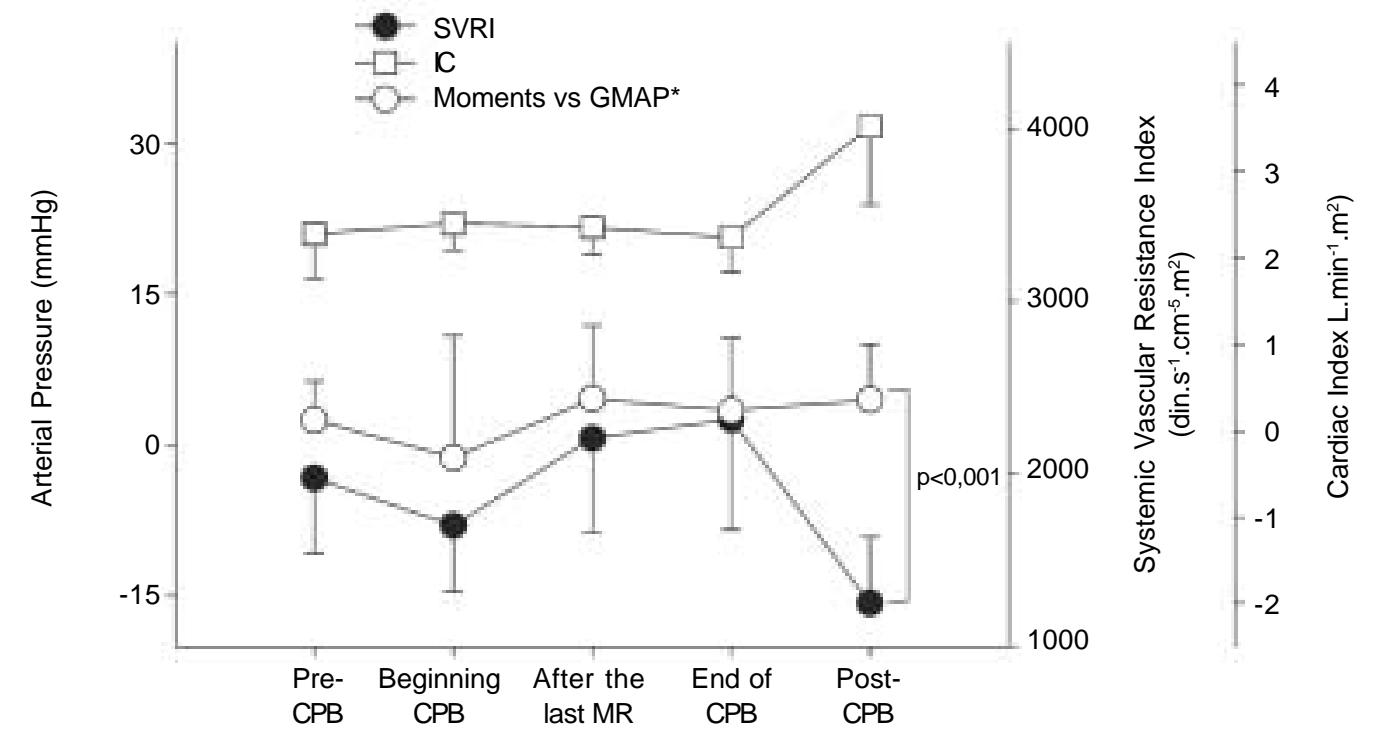

Figure 3 - Temporal Behavior of the Aorta-to-Radial Artery Mean Pressure Gradient (open circles), Systemic Vascular Resistance Index (closed circles), and Cardiac Index (open squares). *GMAP - gradient of mean arterial pressure. 


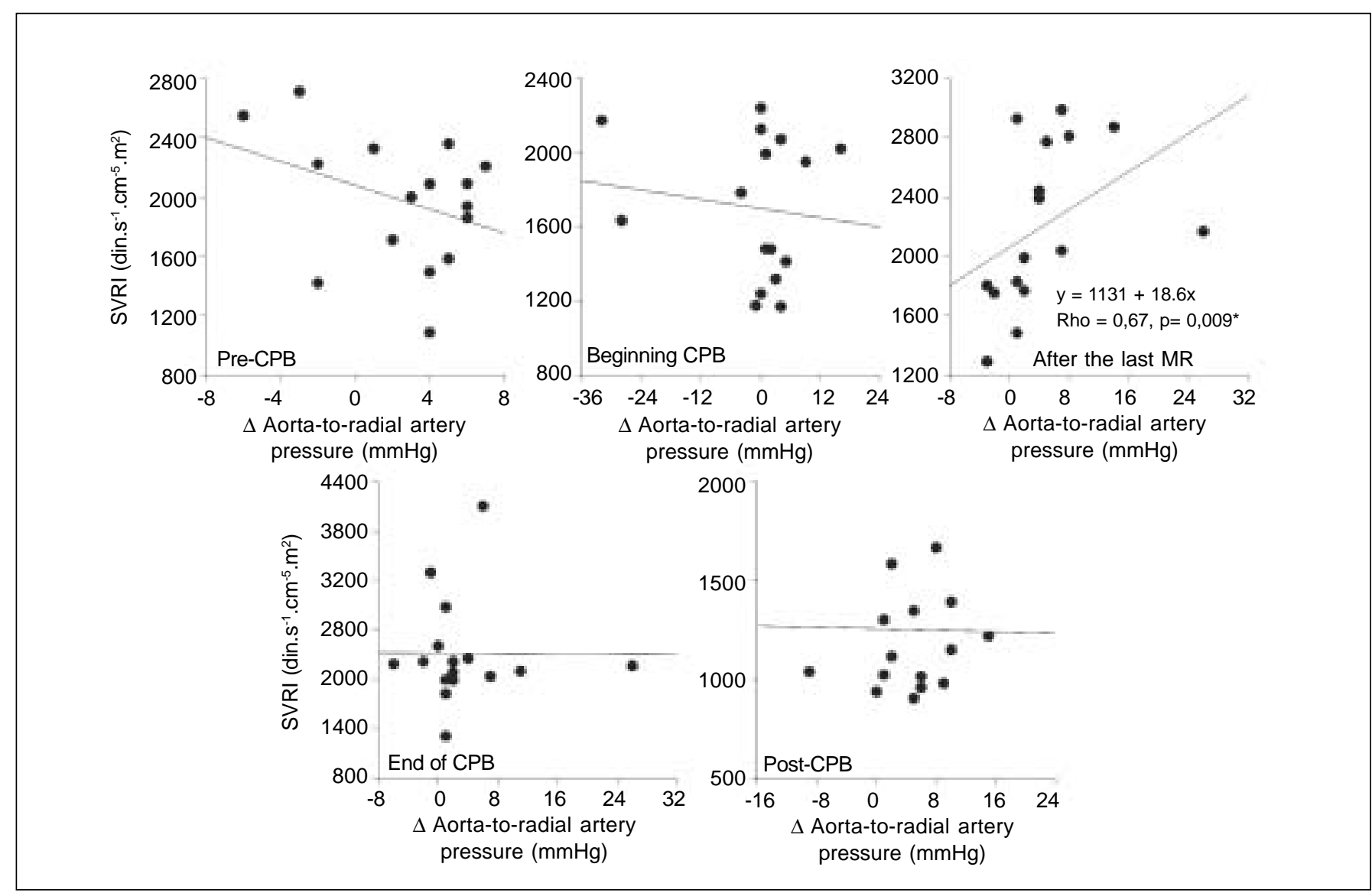

Figure 4 - Dispersion Graphic Evaluating the Correlation Between the Systemic Vascular Resistance Index and the Aorta-to-Radial Artery Mean Pressure Gradient pre-CPB (left upper panel), Beginning CPB (upper center panel), after the Last Myocardial Revascularization (right upper panel), at the End of the CPB (left lower panel), and after Discontinuing the CPB (right lower panel).

\section{DISCUSSION}

The main results of this study were: 1 ) the mean arterial pressure in the radial artery underestimates the arterial pressure at the aortic root after beginning the CPB; however, this difference is approximately 3 to $5 \mathrm{mmHg}$; 2) a consistent correlation between the aorta-to-radial artery pressure gradient and the vascular systemic resistance index after beginning cardiopulmonary bypass was not observed.

Invasive monitoring of the radial artery pressure is routinely done in patients undergoing cardiac surgery with cardiopulmonary bypass, due to the need of real-time control of the perfusion pressure, the catheter is easily inserted and the incidence of complications is low. Based on the radial artery pressure, vasopressors or vasodilators are prescribed to adjust the systemic vascular resistance and to maintain adequate balance between the greater cardiac output possible and the perfusion pressure. In this specific population of patients undergoing cardiac surgery with hypothermic cardiopulmonary bypass, several studies have demonstrated a discrepancy between the radial artery pressure and the pressure at the aortic root ${ }^{6,10-12}$, which could contribute for the inadequate hemodynamic management; this can lead to an inappropriate increase in myocardial oxygen consumption when the radial pressure underestimates the aortic pressure or tissue hypoperfusion in patients whose radial artery pressure is greater than the aortic pressure ${ }^{6,11}$. In this study, it was observed that, after beginning CPB, the mean arterial pressure at the root of the aorta was smaller than in the radial artery by a mean of $1.2 \mathrm{mmHg}$. This gradient was inverted in posterior moments, in which the mean aortic pressure was 3 to $5 \mathrm{mmHg}$ greater than the mean radial artery pressure. In situations in which high concentrations of vasopressors are needed, this gradient might be even greater.

Due to the lack of accuracy of the pressure measured in the radial artery to reflect the pressure at the root of the aorta, other indices, such as systemic vascular resistance could, in theory, provide estimates of the degree of difference between the aortic and radial artery pressures during and after the hypothermic phase of the CPB and provide more adequate evaluations for the hemodynamic adjustments necessary. Despite the temporal behavior of the SVRI being in agreement with the aorta-to-radial artery pressure gradient in most moments, as can be observed in figure 3 , the 
correlation between the systemic vascular resistance and the aorta-to-radial artery pressure gradient was observed only after the last anastomosis in the myocardium, when the patient was being rewarmed and receiving vasoactive drugs. The systemic vascular resistance calculated through invasive hemodynamic measurements at the bedside with intravascular catheters is computed as the ratio between the radial artery-to-right atrium pressure gradient and the systemic flow, reflecting mainly the impedance promoted by the arteriolar microcirculation and the venous bed to the blood flow. The absence of correlation suggests that other factors besides the resistive arteriolar and venous components might contribute to the genesis of the aorta-to-radial artery pressure gradient. However, besides this resistive microvascular circulatory component after beginning CPB, other factors also seem to be related with the modulation of pressure transmission in the arteries, which does not allow interferences from the magnitude of the aorta-to-radial artery pressure gradient from the SVRI.

Several mechanisms have been proposed to explain the aorta-to-radial artery pressure gradient in patients undergoing CPB, and hypothermia, hemodynamic instability, change from pulsatile to continuous non-pulsatile blood flow, and the variation in the caliber of the great vessels due to a change in arterial elasticity induced by the CPB or by high doses of vasomodulator drugs are mentioned more often. Immediately after beginning CPB, the radial artery pressure was slightly higher than the pressure in the aortic root. At this moment there is severe hemodilution due to the 2 liters of Ringer's lactate added to the circulation, causing an abrupt reduction in hematocrit and consequent reduction in blood viscosity and systemic vascular resistance ${ }^{13}$, which could compensate the vasoconstriction induced by hypothermia and release of inflammatory mediators during CPB ${ }^{6,7}$. During the $\mathrm{CPB}$, great part of the crystalloid in the perfusate leaks to the extravascular space as a consequence of the systemic inflammatory reaction induced by the CPB ${ }^{14}$, which might increase the hematocrit and blood viscosity, contributing to the increase in vascular resistance in the smaller arteries, which would justify the beginning and maintenance of the aorta-to-radial artery pressure gradient at 3 to $5 \mathrm{mmHg}$, even after discontinuation of the CPB ${ }^{16}$. On the other hand, De Hert et al. did not observe a correlation between the magnitude of the pressure gradient and the changes in the hematocrit and blood temperature in 68 patients ${ }^{7}$. Another factor possibly associated with the genesis of the aorta-toradial artery pressure gradient is the caliber of the large arteries. Studying 12 patients undergoing cardiac surgery with CPB, Kanazawa et al. observed that 7 patients had an aortato-radial artery pressure gradient greater than $10 \mathrm{mmHg}$ after CPB ${ }^{12}$. This reduction in radial artery pressure in relation to the aortic pressure was associated with a progressive reduction in the velocity of the pulse wave towards the periphery. Using the equation proposed by Moens-Korteweg to calculate the arterial elasticity based on the velocity of the pulse wave ${ }^{16}$, those authors observed a reduction in elasticity that could lead to a dampening of the pulse wave and of the arterial pressure at the level of the radial artery, thus explaining the aorta-to-radial artery pressure gradient ${ }^{12}$. This change in vascular tone could be secondary to the release of vasoactive substances, commonly observed after beginning CPB ${ }^{14}$. Finally, anatomical changes, such as arterial atheromas, could contribute to the reduction in radial artery pressure in relation to the aortic pressure. Due to the inversion of the pressure gradient immediately after beginning CPB, suggesting the absence of arterial stenosis, the presence of anatomical alterations does not seem to be an important contributing factor for the genesis of the aorta-to-radial artery pressure gradient in the present study. In cases in which the condition of the radial artery might be an important factor in the genesis of the pressure gradient, monitoring could be done by puncturing the brachial or femoral artery ${ }^{17}$.

To conclude, the radial pressure underestimated systematically the pressure at the root of the aorta after the beginning of hypothermic cardiopulmonary bypass in patients undergoing myocardial revascularization and this pressure gradient was between 3 and $5 \mathrm{mmHg}$. Although small, this difference can lead to inappropriate or even harmful management conducts. Systemic vascular resistance does not provide an accurate estimate of the magnitude of the aorta-to-radial artery pressure gradient, and this difference of 3 to $5 \mathrm{mmHg}$ between the aortic and radial artery pressures should be considered when making therapeutic decisions in patients undergoing myocardial revascularization with hypothermic CPB.

\section{REFERÊNCIAS - REFERENCES}

01. Pauca AL, Hudspeth AS, Wallenhaupt SL et al. - Radial arteryto-aorta pressure difference after discontinuation of cardiopulmonary bypass. Anesthesiology, 1989;70:935-941.

02. Pauca AL, Wallenhaupt SL, Kon ND - Reliability of the radial arterial pressure during anesthesia. Is wrist compression a possible diagnostic test? Chest, 1994;105:69-75.

03. Maruyama K, Horiguchi R, Hashimoto $\mathrm{H}$ et al. - Effect of combined infusion of nitroglycerin and nicardipine on femoral-to-radial arterial pressure gradient after cardiopulmonary bypass. Anesth Analg, 1990;70:428-432.

04. Baba T, Goto T, Yoshitake A et al. - Radial artery diameter decreases with increased femoral to radial arterial pressure gradient during cardiopulmonary bypass. Anesth Analg, 1997;85: 252-258.

05. Nakayama R, Goto T, Kukita I et al. - Sustained effects of plasma norepinephrine levels on femoral-radial pressure gradient after cardiopulmonary bypass. J Anesth, 1993;7:8-16.

06. Rich GF, Lubanski RE Jr, McLoughlin TM - Differences between aortic and radial artery pressure associated with cardiopulmonary bypass. Anesthesiology, 1992;77:63-66.

07. De Hert SG, Vermeyen KM, Moens MM et al. - Central-toperipheral arterial pressure gradient during cardiopulmonary bypass: relation to pre- and intra-operative data and effects of vasoactive agents. Acta Anaesthesiol Scand, 1994;38:479-485.

08. Ganz W, Donoso R, Marcus HS et al. - A new technique for measurement of cardiac output by thermodilution in man. Am J Cardiol, 1971;27:392-396. 


\section{EVALUATION OF THE AORTA-TO-RADIAL ARTERY PRESSURE GRADIENT IN PATIENTS UNDERGOING SURGERY WITH CARDIOPULMONARY BYPASS}

09. Swan HJ, Ganz W - Variability between measurements of cardiac output. Crit Care Med, 1976;4:279-280.

10. Stern DH, Gerson JI, Allen FB et al. - Can we trust the direct radial artery pressure immediately following cardiopulmonary bypass? Anesthesiology, 1985;62:557-561.

11. Pauca AL, Wallenhaupt SL, Kon ND et al. - Does radial artery pressure accurately reflect aortic pressure? Chest, 1992;102: 1193-1198.

12. Kanazawa M, Fukuyama $\mathrm{H}$, Kinefuchi $\mathrm{Y}$ et al. - Relationship between aortic-to-radial arterial pressure gradient after cardiopulmonary bypass and changes in arterial elasticity. Anesthesiology, 2003;99:48-53.

13. Otsuki DA, Cardieri FA, Schmidt AP et al. - Comparison between intermittent and continuous measurement of cardiac output after acute normovolemic hemodilution in pigs. Artif Organs, 2006;30:458-466.

14. Wan S, LeClerc JL, Vincent $\mathrm{JL}$ - Inflammatory response to cardiopulmonary bypass: mechanisms involved and possible therapeutic strategies. Chest, 1997;112:676-692.

15. Goda Y, Takita K, Gando S et al. - Hemodilution has an important role in femoral-to-radial artery pressure gradient after cardiopulmonary bypass. Circ Cont, 1995;16:223-228.

16. Hamilton WF, Remington JW, Dow $P$ - The determination of the propagation velocity of the arterial pulse wave. Am J Physiol, 1945; 144:521-535.

17. Mohr R, Lavee J, Goor DA - Inaccuracy of radial artery pressure measurement after cardiac operations. J Thorac Cardiovasc Surg, 1987;94:286-290.

\section{RESUMEN}

Carmona MJC, Barboza Jr LCM, Buscatti RY, Gaiotto FA, Hueb AC, Malbouisson LMS - Evaluación del Gradiente Presórico Aorto-Radial en Pacientes Sometidos a la Intervención Quirúrgica con Circulación Extracorpórea.
JUSTIFICATIVA Y OBJETIVOS: Diversos estudios han demostrado diferencia significativa entre la presión aórtica y la presión radial en pacientes sometidos a la circulación extracorpórea (CEC). Los objetivos de este estudio fueron evaluar el comportamiento de la diferencia entre presión arterial radial y la presión aórtica durante revascularización del miocardio (RM) con CEC y su correlación con la resistencia vascular sistémica.

MÉTODO: Después de la aprobación por el Comité de Ética hospitalaria, 16 pacientes sometidos a la RM con CEC hipotérmica fueron estudiados. Presiones sistólica, diastólica y media fueron obtenidas en la raíz de la aorta y en la arteria radial, a través de catéteres específicos. Débito cardíaco se obtuvo usando catéter de arteria pulmonar o directamente de la máquina de CEC y Resistencia Vascular Sistémica indexada (RVSi) fue calculada en los momentos pre-CEC, inicio de la CEC, después de la última $R M$, al final de la CEC y pos-CEC. El análisis estadístico se realizó a través de Análisis de Variancia para medidas repetidas y correlación de orden de Spearman y el nivel de significancia se estableció en 0,05 .

RESULTADOS: Después del inicio de la CEC, la presión arterial radial fue sistemáticamente menor que la presión aórtica en 3 a 5 $\mathrm{mmHg}$. Se observó correlación significativa entre el gradiente medio de presión aorto-radial y la RVSi solamente después de la ultima RM, correspondiendo al calentamiento del paciente ( $R$ ho $=$ $0,67, p=0,009$ ).

CONCLUSIONES: La medida de presión en la arterial radial subestimó sistemáticamente la presión arterial en la raíz de la aorta después de la CEC y la RVSi no suministró estimación puntual de la magnitud del gradiente de presión aorto-radial. 